\title{
Excavación de galerías en rocas duras mediante máquina tuneladora (minador continuo)
}

G. Víé, ingeniero de minas 579-19

sinopsis

Se estudian en este artículo las características más sobresalientes de las máquinas tuneladoras continuas, así como las de sus herramientas de perforación, que proporcionan para las rocas de gran dureza un ahorro sobre los métodos de martillo y explosivo entre el 30 y el 50 por 100.

Se ve la evolución sufrida por estas máquinas que nos ha llevado hasta la muela de disco simple, el rodillo cónico, la corona de perforación de diámetro - aproximadamente la mitad de la galería excavada-, el paso en espiral en sustitución del circular, etc. que proporcionan una serie de ventajas sobre las anteriores.

La excavación continua de una galería de gran sección, mediante tuneladoras o excavadoras continuas (tipo topo), se ha generalizado en todo el mundo.

El efecto directo de una máquina de este tipo es provocar la rotura de la roca en el frente de avance, transformándola en fragmentos que puedan ser evacuados por un sistema automático de transporte. (Desescombro).

El "minador continuo" puede resumirse a dos elementos esenciales: el dispositivo de corte que llevan las herramientas en contacto directo con el terreno frontal, y el mecanismo de evacuación de los fragmentos que resultan de este ataque.

Las herramientas son: o picos de acero revestidos de carburo de tungsteno usados para las formaciones litológicas blandas o de una dureza relativamente débil, o bien discos o muelas. Las muelas derivan del tricono de las perforaciones petrolíferas.

Los discos y muelas van instalados sobre rodillos que giran locos sobre la roca.

Los discos pueden ser simples o dentados, o provistos de pastillas de carburo de tungsteno para resistir mejor la abrasión.

Los rodillos son ensambladuras de elementos cónicos o cilíndricos.

La cabeza cortadora es una corona de diámetro casi igual al de la galería excavada, que gira como una sola pieza. Sobre esta corona se fijan las herramientas de perforación.

Todas las máquinas que excavan a sección total, y particularmente las de muchas muelas, exigen fuertes presiones axiles sobre el frente de avance. Por esta razón, el avance es un poco intermitente, ya que es preciso al fin de la carrera de los gatos hidráulicos devolver su pistón respectivo a la posición cero para poder volver a empujar.

1.-Cabeza de perforación; 2.-Puntos de apoyo; 3.-Empuje; 4.-Sustentación; 5.-Evacuación de escombros; 6.-Motores.

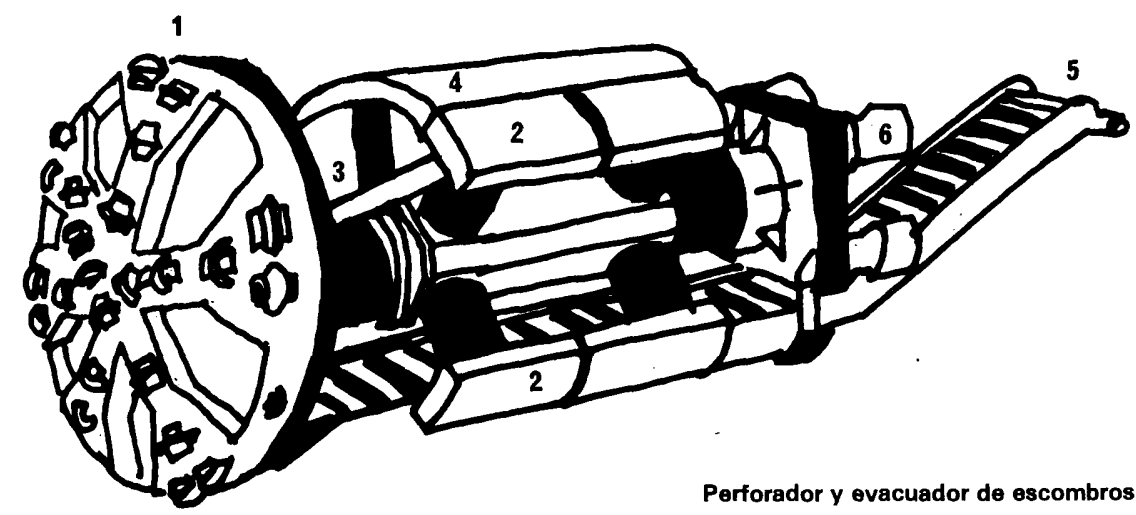


Esos gatos son de doble efecto y permiten el avance mediante propulsión de la máquina en la galería.

Los gatos de presión se fijan a un grupo de apoyo arriostrado contra las paredes del túnel, donde se obtiene la reacción a los esfuerzos desarrollados por el trabajo de los útiles.

La presión ejercida para asegurar el ataque, así como el avance, es función de la dureza de los terrenos encontrados.

El sistema de evacuación de los fragmentos de roca producidos por el trabajo de las herramientas viene determinado por la granulometria de dichos fragmentos. Estos fragmentos no pasan habitualmente del calibre 0-25 o incluso $0-20 \mathrm{~cm}$.

Estos materiales fragmentados se pueden fácilmente recoger mediante un dispositivo de cucharas, evacuándose por cinta transportadora o por cadena a unas vagonetas situadas detrás de la máquina.

Cuando la máquina perfora a sección total una sección de gran diámetro el guiado se regula por laser.

Un obstáculo para la utilización de la excavadora continua es la existencia de una gran dureza en los terrenos durante un tramo de gran longitud.

Se han hecho estudios muy profundos para conocer mejor el trabajo de los discos y muelas. EI empleo de rodillos cónicos evita el desgaste por patinaje de la muela interior, inconveniente que si existe en los rodillos cilíndricos. Esa es la razón por la que las rodaduras de las muelas son oblicuas con respecto al esfuerzo de presión. De esta manera las condiciones de trabajo son más severas.

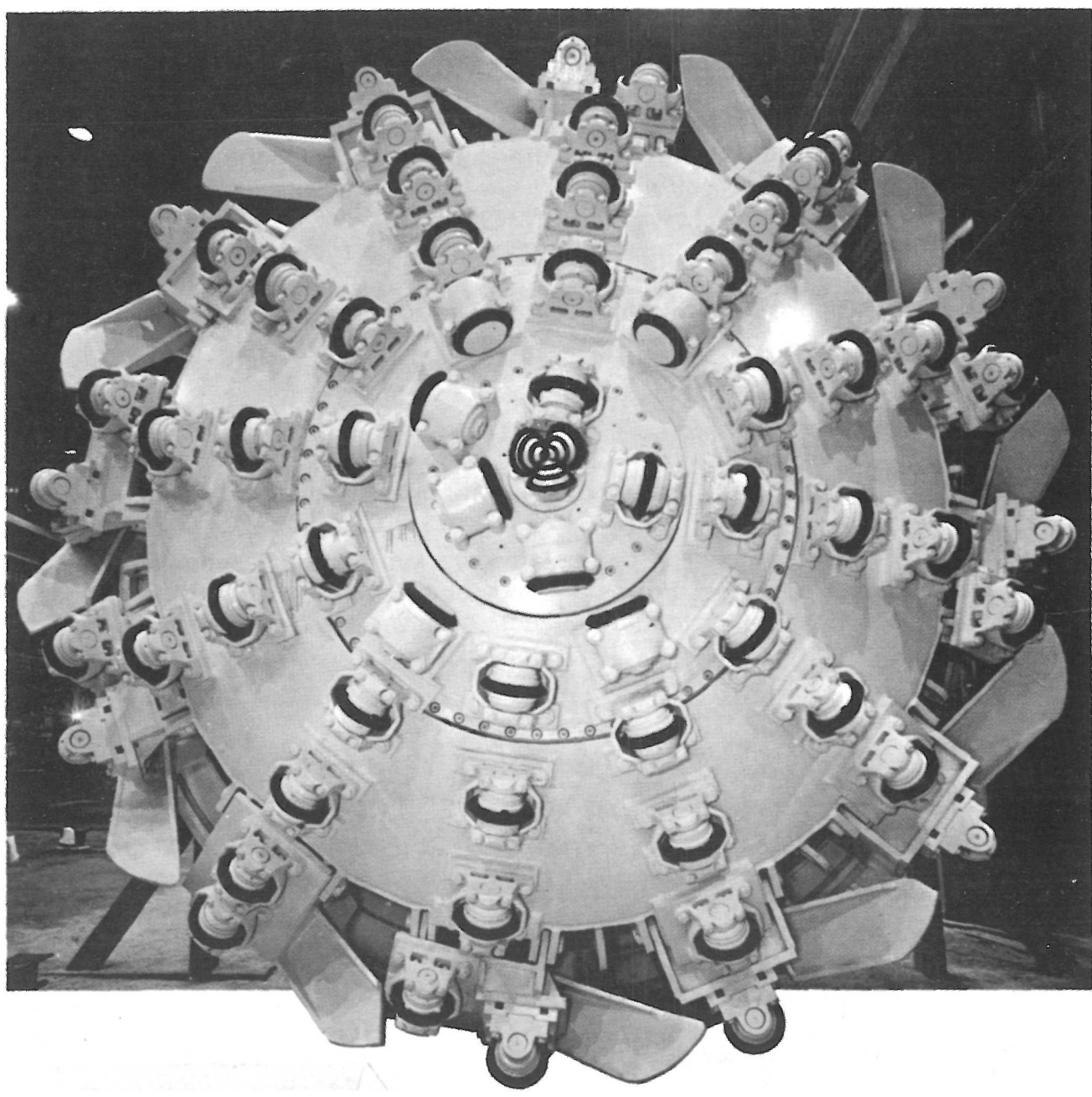

Disco de ataque y de perforación donde se ve la disposición de las muelas. 
Al igual que ocurrió en la perforación petrolífera, con el uso del tricono que redujo el costo de las herramientas, los estudios han llevado al empleo de la muela de disco simple, más o menos reforzada.

Para rocas muy resistentes y abrasivas, las herramientas de perforación están reforzadas con pastillas o botones de carburo de tungsteno.

Se han obtenido excelentes resultados con una máquina WIRTH en la zona granítica de Vallorcine, en EMOSSON, y en los gneis graníticos de Gizling, lo que ha hecho que esta máquina sea la más usada en los Alpes.

Un ejemplo: Dos empresas francesas que contrataron conjuntamente a ELECTRICITE de FRANCE, en el año 1978, la excavación de una galeria de 3,6 m de diámetro y $150 \mathrm{~m}$ de longitud para el salto de agua desde Grand-Maison hasta el torrente Eau-d'Olle (Isére) situado aguas abajo a la cota $1.561 \mathrm{~m}$, usaron una máquina excavadora WIRTHH TB II 328/360 con las caracteristicas siguientes:

- Diámetro de la cabeza de perforación: 3,28 a $360 \mathrm{~m}$.

- Potencia de mando: 3 x $132=396 \mathrm{~kW}$.

- Rotación: 0 a 12 vueltas/minuto.

- Par de rotación: $26.000 \mathrm{kp} \times \mathrm{m}$.

- Presión de perforación hasta $440 \mathrm{Mp}$.

- Carrera de perforación: $800 \mathrm{~mm}$.

- Velocidad: 0 a $5 \mathrm{~cm} /$ minuto.

- Presión hidráulica: $225 \mathrm{~kg} / \mathrm{cm}^{2}$.

- Fuerza de apriete: $1.130 \mathrm{Mp}$.

- Potencia total instalada: $460 \mathrm{~kW}$

- Alimentación de alta tensión: $5.500 \mathrm{~V}$.

- Transformadores de una potencia de 2 x 400 kVA.

- Baja tensión: $380 \mathrm{~V}$.

- Frecuencia: $50 \mathrm{~Hz}$.

- Pesos:

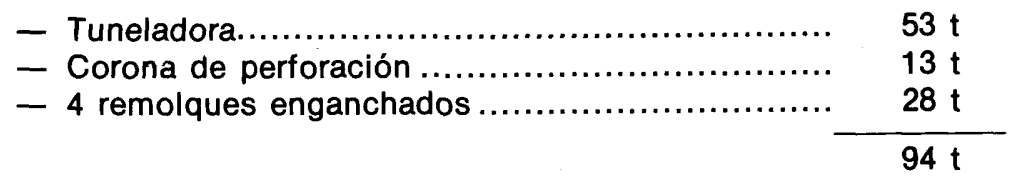

- Longitud total del conjunto $38,50 \mathrm{~m}$.

- La cabeza de perforación está equipada de 20 muelas de doble disco y tiene, además en el centro, dos muelas de piquillos cónicos y dos muelas de piquillos troncocónicos.

- Una desempolvadora se instaló sobre máquina excavadora.

El desescombro se efectuaba por cinta transportadora de $500 \mathrm{~mm}$, que vierten en vagonetas de $8 \mathrm{~m}^{3}$ de capacidad cada una.

El llenado de dos de esas vagonetas corresponde a la evacuación de los fragmentos obtenidos en un avance de $0,80 \mathrm{~m}$.

La excavación empezó el día 8 de agosto de 1978, obteniéndose los siguientes rendimientos:

\section{1.-En el cristalino (gneis)}

$338 \mathrm{~m}$, del 8 de agosto al 8 de septiembre de 1978.

Entre el 10 y el 18 de agosto la perforación se detuvo con objeto de completar las instalaciones de evacuación de los escombros sobre la vía y acondicionar la descarga, 0 sea, 18 dias de trabajo con tres turnos de 8 horas. Media: $19 \mathrm{~m} /$ día.

\section{2. - En el Lias}

$162 \mathrm{~m}$, del 8 al 18 de septiembre de 1978 .

6 dias de trabajo con tres turnos de 8 horas. Media: $20 \mathrm{~m} /$ día. 


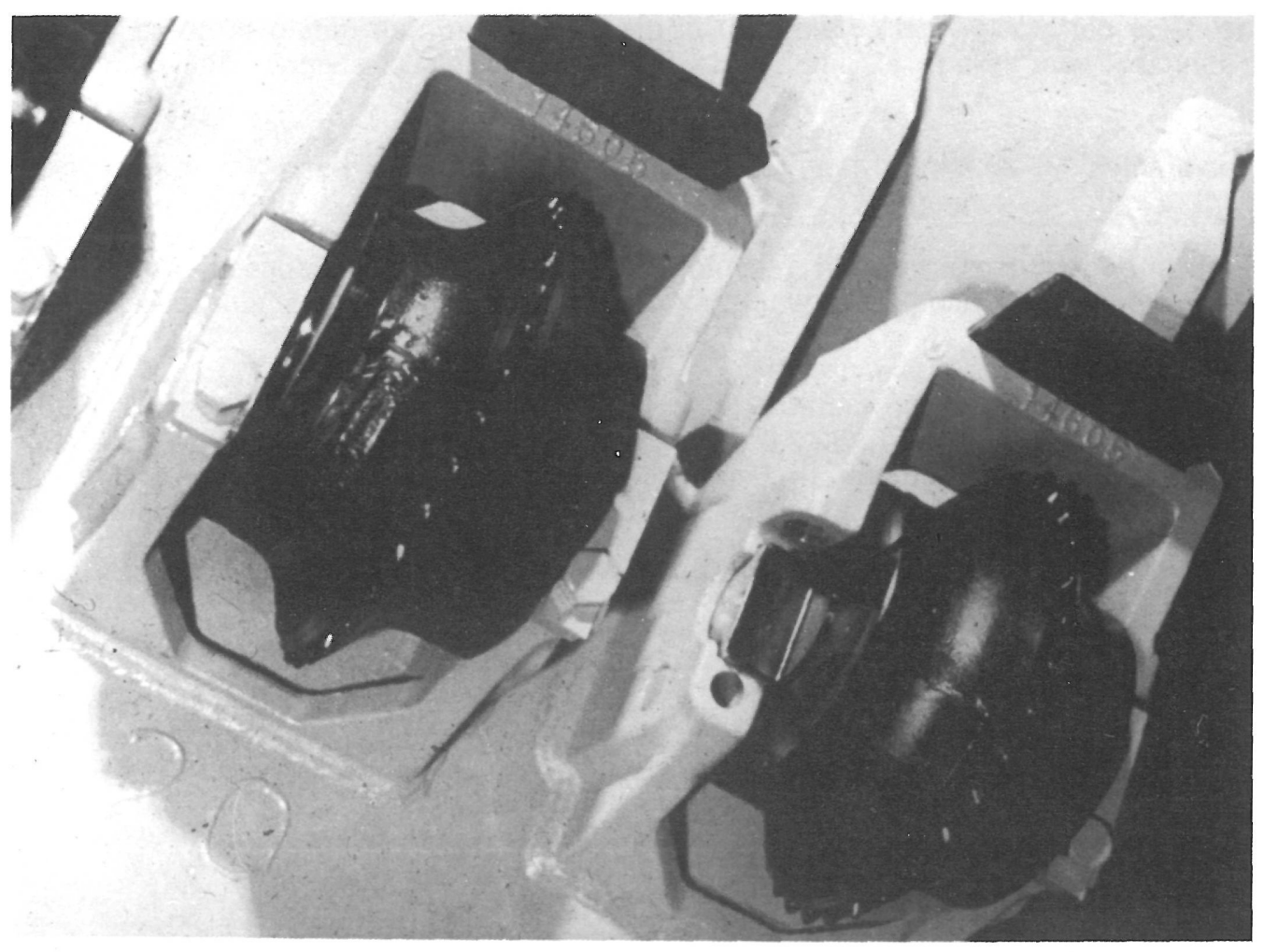

Muelas de disco de carburo de tungsteno para perforar rocas duras.

3. - En el Trias (una corriente de agua de alrededor de $50 \mathrm{l} / \mathrm{seg}$ se encontró en el cambio de roca).

$38 \mathrm{~m}$, del 18 al 20 de septiembre de 1978. Media: $19 \mathrm{~m} /$ día.

\section{4.--En el cristalino (esquistos verdes)}

$310 \mathrm{~m}$, del 20 de septiembre al 18 de octubre de 1978.

18 días de trabajo con tres turnos de 8 horas. Media: $17 \mathrm{~m} /$ día.

Se necesitaron once posiciones de laser para el guiado de la máquina debido a la existencia de una curva de $252 \mathrm{~m}$ de longitud y de $220 \mathrm{~m}$ de radio a la salida de esta galería.

He aquí otro ejemplo de una excavadora continua WIRTH:

La excavación de una galeria forzada de $20 \mathrm{~km}$ de longitud entre el Río Chixoy y el Río Quixal por la firma Hochtief-Essen para los trabajos de acondicionamiento de la central eléctrica Pueblo Viejo Quixal en Guatemala. Las características esenciales del proyecto se pueden resumir así:

- Longitud: $26 \mathrm{~km}$

- Diámetro de la galería: 5,64 m.

- Terreno inútil: más del $1.000 \mathrm{~m}$.

- Formaciones geológicas más importantes que hubo que perforar: anhidrita, gres, esquisto, roca caliza, dolomita, marga.

- Resistencia a la compresión, hasta $1.200 \mathrm{~kg} / \mathrm{cm}^{2}$ con un 55 por 100 de sílice.

Para este trabajo se usaron dos máquinas WIRTH, del tipo TB-V, que perforan con un diámetro de $5,64 \mathrm{~m}$. Estas máquinas se distinguen de las características "standards" por tener una conicidad reducida en la corona de perforación, con el acondicionamiento de una especie de faldón para ella y con un tren posterior para facilitar el avance de la máquina a medida que se progresa en la perforación. 


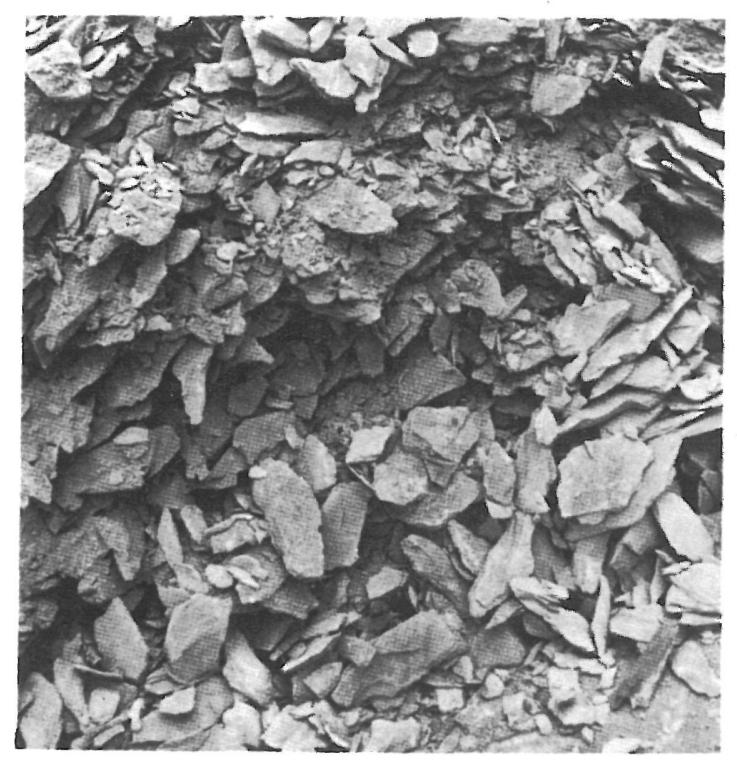

Escombros de rocas cuyo corte ha sido efectuado con discos y muelas

El gato al final de cada barrido, bien sea hacia el interior, bien sea hacia el exterior, se para, y entonces gracias a la intervención de los gatos de presión, el gato avanza una cierta longitud, que varía de 5 a $30 \mathrm{~mm}$, según los terrenos.

Esta penetración, o avance, se realiza en un espacio muy corto de tiempo, el necesario para un giro de la cabeza.

Después de producirse el avance, el gato de barrido entra en acción de nuevo, y las herramientas comienzan otra vez su ataque frontal, quitando un trozo de terreno de espesor igual al de la penetración.

Es evidente que durante la perforación en "sentido estricto" la máquina está inmovilizada y sólo la corona de perforación da vueltas y los brazos barren.

De esta manera, estamos ante una máquina "tuneladora" simplificada, que consta de un cuerpo cilíndrico y de dos prolongaciones por la parte trasera.

La cabeza fija soporta por su parte delantera, gracias a una serie de rodamientos y estribos, una cabeza móvil, que lleva en su parte trasera el gato de barrido, y por delante los brazos de barrido montados sobre cojinetes y unidos por bielas a dicho gato.

Es precisamente en la cabeza fija donde están alojados los motorreductores de arrastre en rotación de la cabeza móvil.

Para una máquina como la TB-270, las características son, por ejemplo:

- Diámetro de perforación.

$2,90 \mathrm{~m}$

- Potencia total instalada. $260 \mathrm{CV}$

- Potencia utilizada para:

- la cabeza $210 \mathrm{CV}$

- el barrido.

$20 \mathrm{CV}$

- el transporte.

$20 \mathrm{CV}$

- diversos.

$10 \mathrm{CV}$

- Número de útiles..................................... 3

- Velocidad de rotación de la cabeza................ 12 vueltas/minuto

- Presión de la máquina $(3 \times 40)$

$12 \mathrm{v}$

- Par máximo sobre la corona de perforación

$13 \mathrm{t} / \mathrm{m}$

- Tensión de alimentación del aparato................ $380 \mathrm{~V}$

- Peso de la máquina ................................. $30 \mathrm{t}$

- Longitud de la máquina........................... 6,5 m

- Peso de la vagoneta hidráulica y transportador... $5 \mathrm{t}$

- Longitud de la vagoneta hidráulica y transportador. $11 \mathrm{~m}$. 
Para una máquina tuneladora, la TB-300 de $3 \mathrm{~m}$ de diámetro de perforación, las características más importantes son:

- Diámetro de perforación

- Potencia total instalada

- Potencia utilizada para:

- la cabeza.

- el barrido.

- el transporte.

- diversos.

- Número de útiles.

- Velocidad de rotación de la cabeza.

- Par máximo sobre la cabeza de perforación

- Presión de la máquina $(3 \times 40)$

- Peso de la máquina

- Longitud de la máquina...

- Peso de la vagoneta hidráulica y transportador... 15

- Longitud de la vagoneta hidráulica y transportador. $30 \mathrm{~m}$

3

$120 \mathrm{t}$

$35 \mathrm{t}$

$\mathrm{t}$
$3 \mathrm{~m}$

$550 \mathrm{CV}$

$400 \mathrm{CV}$

$60 \mathrm{CV}$

$60 \mathrm{CV}$

$30 \mathrm{CV}$

3 a 42 vueltas/minuto

$25 \mathrm{t} / \mathrm{m}$

$5,5 \mathrm{~m}$

En esta máquina se encuentran disposiciones ya existentes en las anteriores para los mismos fines.

La cabeza fija lleva en su parte inferior delantera una zapata deslizante de sección circular que sirve de apoyo a la máquina por delante.

Las prolongaciones de la parte de atrás constituidas por elementos metálicos unidos, deslizan por una corredera articulada, sirviendo para apoyo trasero de la máquina y llevan tres gatos de presión que permiten el avance de ésta.

Un transportador, que lleva una cadena auto-cargadora, recoge los escombros de la base de la galería y los evacua.

A pesar del limitado número de útiles, la potencia es del mismo orden que la instalada en los tuneladores considerados hasta ahora como clásicos.

Sin embargo la presión ejercida por cada herramienta, de alrededor de $40 \mathrm{t}$, es de cuatro a dos veces la de las máquinas clásicas (10 a 20 t) y la velocidad de rotación es de 40 vueltas/minuto contra las 8 vueltas/minuto de las máquinas antiguas. Esto nos lleva a una potencia de más de $200 \mathrm{CV}$ por cada herramienta, sin calentamientos anormales, ni excesivos desgastes.

Tal instalación deja entrever nuevas posibilidades y mejores resultados para los próximos años.

Ciertamente, el conjunto de los brazos oscilantes del gato de barrido y de las bielas de unión, añade una complicación mecánica suplementaria a la construcción clásica pero, en contrapartida, debe reconocerse que aunque el esfuerzo de presión por herramienta es mayor, la presión total de la máquina es menor, casi del 50 por 100 inferior a la de una máquina clásica.

Esta ventaja es tanto más manifiesta cuando se sabe que el dimensionamiento de una máquina tuneladora es, en un 80 por 100 , función del valor de dicha presión.

Una importante reducción de ésta se traduce en una reducción de las dimensiones y del peso (cerca del 50 por 100).

Esta reducción de peso, lo mismo que el montaje de las herramientas en el extremo de los brazos, permite reducir la importancia del cuerpo de máquina con respecto al túnel excavado, resultando el diámetro de ese cuerpo de alrededor de la mitad del diámetro de la sección excavada. $O$ sea, se obtiene facilidad de montaje, de maniobra y de evacuación de escombros. Además tiene una mejor accesibilidad, una mejor visibilidad y ofrece grandes ventajas en cuanto al barrido. EI paso de barrido es equivalente al paso de las máquinas clásicas.

En la nueva máquina tuneladora Bouygues el movimiento de oscilación de los brazos es accionada mediante un gato. La velocidad de su desplazamiento se puede regular graduando el caudal de alimentación de aceite.

De esta manera el paso de espiral puede adaptarse mejor a la naturaleza de las formaciones atravesadas. 
La ejecución reciente de trabajos de excavación de galerias, con estas máquinas tuneladoras, ha permitido comparar sus interesantes rendimientos con los de otras máquinas usadas para los mismos fines.

Veamos un ejemplo: La excavación de galerías con una longitud total de $18 \mathrm{~km}$ - de ellos $16.900 \mathrm{~m}$, con un diámetro de $2,90 \mathrm{~m}$; y $900 \mathrm{~m}$, con un diámetro de $5,50 \mathrm{~m}$ - realizadas para la obtención de agua en Siria, atravesaban geológicamente dos anticlinales de calizas cretáceas - separados por un sinclinal terciario-, con predominio de conglomerados alrededor de $3.000 \mathrm{~m}$ y de roca caliza en 2.700 metros.

Mientras que la resistencia a la compresión de las pudingas macizas y bien cimentadas era del orden de $20.000 \mathrm{kn} / \mathrm{m}^{2}$, la de las calizas era superior a $60.000 \mathrm{kn} / \mathrm{m}^{2}$ pudiendo, puntualmente, elevarse al doble este último valor.

El resto del trazado pasaba por formaciones geológicas más blandas: calizas y margas cretáceas, arcillas y margas rojas con cantos rodados o, localmente, homogéneas.

Análogamente, algunos conglomerados sin dureza notable fueron igualmente atravesados por la máquina.

Al contrario, en uno de los ataques con pendiente en declive se encontró un conglomerado muy macizo, un cemento siliceo extremadamente duro, de una manera inesperada.

La resistencia de esos elementos a la compresión era localmente superior a $200.000 \mathrm{kn} / \mathrm{m}^{2} \mathrm{para}$ los cantos rodados, y a $100.000 \mathrm{kn} / \mathrm{m}^{2}$ para su cimiento.

A pesar de esas condiciones se mantuvo el avance entre los 7 y $10 \mathrm{~m}$ por día, perforando en tres turnos.

Sin embargo es evidente que para tales pudingas no presenta ventaja la máquina estudiada.

A partir de una de las ventanas intermedias, la máquina debió atravesar margas blandas más o menos impregnadas de agua, llegándose a conseguir un avance instantáneo de 3 a $4 \mathrm{~m} /$ hora, con la contrapartida de un entumecimiento y un esponjamiento de las arcillas en la solera, con lo que se crean algunas complicaciones para el comportamiento correcto de la vía de servicio de la obra.

Los rendimientos en esta zona variaban entre 6 y $28 \mathrm{~m} /$ dia, siendo el mínimo atribuible a la existencia en el frente de apreciables avenidas de agua.

Debido a la gran longitud total del terreno que hubo que perforarse utilizaron dos máquinas Bouygues, con dos ataques ascendentes diferentes a través de las formaciones más heterogéneas, excavando en 25 meses y medio de trabajo $5.894 \mathrm{~m}$, con un diámetro de $2,90 \mathrm{~m}$.

El avance medio efectivo por hora de funcionamiento de esas máquinas fue de 1,15 $\mathrm{m}$.

Otro ejemplo: En el Macizo Central Francés, a una veintena de kilómetros al Norte de Limoges, se trazaron las galerias de reconocimiento de yacimientos de granito, en el seno de los leucogranitos de los Montes de Ambazac.

Una máquina tuneladora muy parecida a las anteriores excava en sección circular de $3 \mathrm{~m}$ de diámetro, o sea, de $7 \mathrm{~m}^{2}$ de sección, con curvas de $40 \mathrm{~m}$ de radio. 
Si la excavación se hubiese hecho con martillo perforador o jumbo de perforación, el granito habria necesitado un gasto de explosivo de goma-dinamita BAM, de alrededor de $2.500 \mathrm{~g} / \mathrm{m}^{3}$, y una barrena de acero con la boca de carburo $\varnothing 33$ para cada $30 \mathrm{~m}^{3}$.

En el curso de los tres primeros meses de ensayo de la máquina, la experiencia ha mostrado que el avance en un mismo granito es de $2,80 \mathrm{~m}$, con un consumo eléctrico del orden de $700 \mathrm{kWh} / \mathrm{m}^{2}$.

La ventaja de la excavación en sección circular es suprimir todo sostenimiento por cercos, bulones, etc., en las travesias difíciles (fallas, por ejemplo).

El pilotaje por laser ha actuado siempre de manera perfecta, especialmente en las curvas.

Por todo esto, el temor que se tenía hasta ahora de que un minador continuo no funcionara eficazmente en una roca de gran dureza, se debe eliminar en presencia de esos primeros resultados, que dejan entrever otros aún mejores, una vez se pongan a punto útiles menos sensibles a la abrasión.

Por consiguiente, el precio del metro de la galería realizado con una máquina de este tipo puede llegar a ser del orden del 30 por 100 al 50 por 100 inferior al obtenido por avance con martillo y con explosivo.

\section{résumé}

\section{CREUSEMENT AU TUNNELIER (MINEUR CONTINU) DE GALERIES EN ROCHES DURES}

\section{G. Vié, ingénieur des mines}

Dans cet article sont étudiées les caractéristiques les plus remarquables des engins tunneliers, ainsi que celles de leurs outils de forage, qui assurent pour les roches très dures une économie sur les méthodes de forage res une économie sur les méthodes de forage
au marteau et à l'explosif entre $30 \%$ et $50 \%$.

On constate que ces engins ont tellement évolué que leurs éléments vont de la molette à disque simple, au rouleau conique, à la tête de forage à diamètre équivalent approtête de forage à diamétre equivalent approximativement à la moitié de la galerie
creusee, et que les outils se déplacent, sur le front d'attaque, en spirales croissantes, ou décroissantes, au lieu de cercles, ce qui permet une série d'avantages sur les engins primitifs.

\section{summary \\ HARD ROCK GALLERY EXCAVATION WITH CONTINOUS BORING MACHINES}

\section{G. Vié, Mining Engineer}

This article studies the most outstanding characteristics of continous boring machines and their drilling tools, giving in hard rock ex cavation a sizable economy of 30 to $50 \%$ relative to the hammering and blasting techniques.

The paper goes through the development of these machines from the simple grinding wheel, to the conical roller bit, the excavating crown with an approximate diameter half of the gallery being excavated, the spiral pitch instead of the circular pitch, etc. each providing a number of advantages over the preceding step.

\section{zusammenfassung}

AUSBRUCH VON STOLLEN IN HARTEN GESTEINEN MITTELS EINER TUNNELBAUMASCHINE

(Daueruntergraber)

G. Vi6, Bergbauingenieur

In diesem Artikel werden die hervorragendsten Merkmale der Tunnelbaumaschinen sowie die inrer Bohrwerkzeuge untersucht, welche bei Gesteinen grosser Härte gegenüber den Hammer- und Sprengmethoden eine Ersparnis von 30 bis $50 \%$ ermöglichen.

Es wird die Entwicklung dieser Maschinen dargestellt, welche bis zur einfachen Schleifscheibe, Kegelwalze, Bohrkrone mit einem Durchmesser von ca. der Hälfte des ausgegrabenen Stollens, Spiraidurchgang anstelle des Runddurchganges usw. führt wobei diese genannten Werkzeuge gegenüber den
bisherigen zahlreiche Vorteile bieten. 